\title{
A atuação do aluno de psicologia no estágio de hospitalar
}

\author{
Graziela Fernanda Verbalen de Freitas* \\ Sue Ellen Ferreira Modesto Rey de Figueiredo** \\ Darklê Ferreira Modesto Barbosa***
}

\begin{abstract}
Resumo
A formação acadêmica tem como finalidade a preparação para a atuação prática. O intuito principal é de que todos os alunos possam chegar, à prática, preparados para a atuação sem que apresentem inseguranças, porém, a ansiedade tende a aumentar decorrente do que a própria situação exige. É um momento em que os acadêmicos assumirão uma postura profissional, nesta ocasião há o processo de construção da identidade profissional e do amadurecimento pessoal e profissional. Diante de tantos estressores, o estagiário terá que desenvolver estratégias de enfrentamento que sejam saudáveis para um melhor aproveitamento do estágio. Além da interlocução da teoria com a prática, há também as inter-relações que o estagiário enfrentará durante esse percurso. Decorrente disso, para o seu crescimento pessoal e profissional é importante que ele tenha um ambiente acolhedor e propício, que deverá ser proporcionado tanto pelo espaço físico quanto pelos profissionais que estão a sua volta. Mas, mais importante neste momento é que o ambiente da supervisão seja um espaço favorável para a aprendizagem.
\end{abstract}

Palavras-chave: psicologia; manejo; prática profissional

\section{The performance of the psychology student at the hospital stage}

\begin{abstract}
The academic qualification is aimed at preparing the students for their professional practical work. Its main goal is to assure that all the students take their professional practice without presenting insecurities, however, the anxiety tends to increase due to the circumstances requires. It is a time that the students will take a professional attitude, on this occasion emerges the process of their professional identity construction, as well as, their personal and professional development. Facing a plenty of stressors, the trainers will have to develop coping healthy strategies for a better procedure at developing their professional stage. Besides of the dialogue between the theory and the practice, there is also the interrelationship between people that the trainee will face along the process. Based on these ideas and due to their personal and professional development, it is important that they come upon a warm and friendly environment. This setting should be provided by both; the physical space and the professionals who are around them. But, more important at the moment is the supervisory environment is a favorable space for learning.
\end{abstract}

Keywords: psychology; handling; professional practice

\footnotetext{
* Psicóloga Clínica, graduada pela Universidade de Cuiabá (UNIC).

** Psicóloga, Doutora em Oncologia pela Fundação Antônio Prudente, Mestre em Psicologia da Saúde pela Universidade Metodista de São Paulo (UMESP), Professora da Faculdade de Psicologia da Universidade de Cuiabá (UNIC) e orientadora de Mestrado em Ambiente e Saúde da Universidade de Cuiabá (UNIC). *** Cirurgiã-Dentista, Doutora em Oncologia pela Fundação Antônio Prudente, Mestre em Clínica Odontológica pela Universidade Vale do Rio Verde (UNINCOR), Professora da faculdade de Odontologia do Centro Universitário de Várzea Grande (UNIVAG).
} 


\section{Introdução}

O presente trabalho visa apresentar uma reflexão a respeito da formação profissional em psicologia com o foco no estágio de psicologia hospitalar, pautado principalmente pela relação supervisor-aluno no que concerne à construção de um ambiente de supervisão favorável ao processo de ensino-aprendizagem.

A vivência estudantil na universidade é uma questão pouco investigada e discutida, especialmente nos cursos de Psicologia, além das realizações durante o processo, existe também o sofrimento que pode acompanhá-la, decorrente do contato com o sofrimento psíquico das pessoas atendidas ou com os conteúdos acadêmicos mais diretamente relacionados com a subjetividade humana (Andrade, Tiraboschi, Antunes, Viana, Zanoto \& Curilla, 2016). Apesar da relevância dos estágios na formação, há uma carência dessa discussão na literatura, principalmente em sua ligação com os cursos de Psicologia (Cury \& Neto,2014).

A supervisão de estágio é, nesse sentido, o espaço proposto a desenvolver nos iniciantes uma postura adequada à sua inserção na profissão. Esse saber-fazer na psicologia, as experiências iniciais e a formação de competências para a prática são compartilhadas entre os membros do grupo de supervisão pelo qual o professor supervisor tem responsabilidade didático-pedagógica (Menezes e Medrado, 2013).

Estudar o processo de formação do psicólogo é importante, principalmente no que tange a teoria e a prática devido ao fato do estágio ser o contato com a experiência profissional após anos de estudos teóricos (Pires, 2011). Constata-se que essa experiência representa, na concepção dos estagiários, um crescimento junto com seu paciente, o que o ajuda a tornar-se terapeuta (Silva, Coelho \& Pontes, 2017).

A formação acadêmica baseia-se na preparação do aluno para a atuação prática, porém, é difícil presumir que todos os alunos estarão seguros de seus aprendizados para o início da atuação prática da sua profissão. Esse fato é muito frequente nos estudantes, quando estes se deparam com os estágios, mas é perceptível também que psicólogos formados que não possuem especialização em psicologia hospitalar também se sintam inseguros, de alguma forma, para iniciar a prática. De acordo com essa linha de pensamento, Tonetto e Gomes (2007) relatam a respeito da dificuldade em definir a ênfase profissional a ser seguida ainda na graduação.

O psicólogo ao iniciar sua prática no ambiente hospitalar se sente hesitante nesse novo ambiente devido a questões diversas, dentre elas o fato do aprendizado teórico na graduação não ser suficiente para diminuir ou suprimir com a insegurança em relação ao atendimento hospitalar. Esse fato se dá devido a gama de conhecimento necessário para se trabalhar num ambiente hospitalar, exigindo assim outras habilidades desse profissional. Novas alterações curriculares veem de encontro à necessidade de melhorar essa base apreendida pelo aluno de psicologia com o intuito de fornecer as habilidades necessárias para a atuação prática de forma eficaz e satisfatória (Torezan, Calheiros, Mandelli \& Stumpf, 2013).

É difícil imaginar a possibilidade de um psicólogo com uma boa formação clínica se adentrar na área da psicologia hospitalar apenas com as habilidades clínicas que ele possuía (Gorayeb, 2010). Diante disso, Tonetto e Gomes (2007), afirmam que o profissional precisa ser capacitado para assistência, ensino e pesquisa. Assistência tanto do micro (o paciente), do meso (familiares) e até do macro (da equipe, dos funcionários), de forma individual ou grupal, com enfoque psicológico ou multidisciplinar.

Isso demonstra que inicialmente o aluno de psicologia deve ter disciplinas que demonstrem a inserção do profissional de psicologia em diversos níveis de atenção à saúde e depois estimular a continuidade do conhecimento em programas de formação específicos (especialização, residência, etc.) da área em questão (Gorayeb, 2010). Diante disso, o Estágio é o momento de encontrar a prática, momento essencial em um processo formativo profissional. O papel do estágio é proporcionar experiências para os estudantes, nas quais as atividades desenvolvidas produzam aprendizagens sobre a Psicologia, seus saberes e fazeres. (Santos \& Nóbrega, 2017).

Verificando-se esta demanda, este artigo tem o intuito de compartilhar noções básicas para o início do estágio em psicologia hospitalar, a fim de auxiliar questões relacionadas a possíveis dúvidas, expectativas, fantasias, idealizações entre outras.

\section{A psicologia hospitalar}

Seja na esfera do serviço público ou nas instituições privadas, a Psicologia ligada à área da Saúde, na realidade brasileira, ainda tem uma história significativamente recente (Santos \& Jacó-Vilela, 2009). No amplo contexto da Psicologia da saúde, a Psicologia hospitalar se caracteriza no Brasil como uma especialidade em notável crescimento e que demanda o exercício de habilidades específicas e variadas dos profissionais (Torezan, Calheiros, Mandelli \& Stumpf, 2013). 
No Brasil, a psicologia Hospitalar ainda apresenta pouca inserção nos hospitais. Tanto nos hospitais públicos quanto privados, os psicólogos existentes não são suficientes para atender a demanda. São Paulo e Brasília são as cidades que mais se destacam quanto a inserção do psicólogo hospitalar nessas instituições, deixando as demais cidades em defasagem (Santos, 2015).

Em sua prática, mesmo fora dos hospitais, e em suas teorias, o psicólogo está acostumado a trabalhar com o adoecer, com os impactos de uma doença orgânica sobre o estado emocional das pessoas; com o tema da morte; entre outros. Assim, muitos desafios com os quais se depara o psicólogo nos hospitais não estavam, até então, ausentes de sua prática clínica nem de suas teorizações (Carvalho \& Sampaio, 1997).

O processo de apropriação da experiência pelos seres humanos ao longo da vida social permite constituir qualidades, capacidades e características humanas, bem como desenvolver continuamente novas aptidões e funções psíquicas (Pires, 2011).

O trabalho num Hospital Geral comporta ao mesmo tempo a essência da multiprofissionalidade e a faceta do corporativismo, a multiplicidade do adoecimento e a singularidade de cada paciente, a Psicologia ancorada em diversas abordagens e a defesa por um campo unitário de atuação. Ao psicólogo que se aventura nesta seara torna-se necessário buscar subsídios para um estilo próprio de atuação (Santos \& Jacó-Vilela, 2009).

Tonetto e Gomes (2007) ressaltam que as habilidades (poder técnico ou legal para realizar uma conduta) e competências (uso eficaz de habilidades para efetuar um ato, ou seja, a escolha de um entre vários procedimentos disponíveis) são exigidas na prática da psicologia hospitalar, desde a formação enquanto estagiário, o qual recebe suporte e direcionamento da formação acadêmica e do supervisor.

\section{O estágio em psicologia hospitalar}

A formação acadêmica em si pode desencadear sentimentos de ansiedade devido à carga emocional presente durante a realização do curso em sim. Nos últimos anos, devido aos estágios que proporcionam o contato com a prática, essa ansiedade tende a aumentar decorrente das exigências que a situação demanda. Neste momento é necessário que os acadêmicos assumam uma postura profissional e façam a articulação da teoria com a prática (Franco, 2001).

A tensão histórica advinda da relação entre teoria e prática persiste. Em determinados contextos a teoria ain- da permanece, para vários docentes como fundamento da prática. Em contrapartida, corre-se atualmente o risco da construção de modos de ação operativos e pragmáticos, sem o necessário apuro conceitual (Cury \& Neto, 2014).

O estágio proporciona a experiência fundamental de entrar em contato com a realidade da profissão, o que faz dele um momento-chave da formação. Constitui circunstâncias plausivelmente representativas da prática profissional - porém, ainda como parte do curso - nas quais o aluno estagiário pode desenvolver competências básicas (teóricas, metodológicas, instrumentais e éticas) para realizar suas atividades práticas e, assim, refletir criticamente sobre a realidade social, educacional, política e econômica do meio social (Pires, 2011). Isso demonstra que o estágio exerce um papel relevante proporcionando experiências que contribuem para a formação em Psicologia e permitem aproximações com a prática profissional (Santos \& Nóbrega, 2017).

Os desafios das intervenções realizadas pelos estagiários refletem o confronto entre teoria e prática na rede de saúde a qual ele se encontra. Esse movimento resulta em buscas de alternativas e estratégias pertinentes com o intuito de superar os impasses e as dificuldades apresentadas nessa nova experiência de preparação profissional (Santos, 2011).

É importante entendermos o enlaçamento necessário entre teoria e prática, favorecendo a sinergia do encontro entre essas duas dimensões da prática acadêmica na direção do aperfeiçoamento da formação (Cury \& Neto, 2014). É por meio do estágio que se oportuniza a articulação da teoria com a prática, pois ao serem inseridas em determinados campos, buscam contextualizar a teoria, as discussões em sala de aula, com a realidade encontrada nas instituições e nas comunidades (Santos \& Nóbrega, 2017).

Diante disso, a aplicação da noção de competências exige relação e integração entre orientador e um projeto pedagógico capaz de causar a igualdade da sala de aula, dando espaço a estágios de qualidade e articulação com o mercado de trabalho. Um excelente plano pedagógico por competências requer cuidadoso sistema de avaliação tanto para o supervisor quanto para os estagiários (Tonetto \& Gomes, 2007).

A prática de um estágio na área da saúde, articulado à formação do aluno, apesar da importância assumida pelos próprios acadêmicos e pelas instituições formadoras, também pode ser fonte de sofrimento e conflitos (Moulin, 1998). É por meio da interação existente que o estagiário vai se apropriar do seu estágio, do fazer do 
psicólogo, aprendendo, colaborando e se apropriando de novos conhecimentos (Santos \& Nóbrega, 2017).

Por mais que os acadêmicos tenham sido preparados durante a faculdade para sua atuação na prática profissional, neste caso na Psicologia Hospitalar, nota-se que ao se deparar com o estágio conseguem perceber a verdadeira dimensão da prática, o que pode fazer com que esse momento seja impactante, frustrante e temido (Guedes, 2006).

Além disso, por conta de diferentes contextos culturais, os estagiários e os pacientes geralmente não compartilham das mesmas expectativas terapêuticas, fator que pode levar ao abandono do tratamento por parte do paciente desencadeando diversas reflexões no estagiário (Comin, Souza, \& Santos, 2008). As primeiras angústias tomam a forma de questões, as quais, ao serem levadas, juntamente com as confusões e os tropeços para supervisão, são clareadas (Silva, Coelho \& Pontes, 2017).

$\mathrm{O}$ medo de errar e a responsabilidade de estar lidando com seres humanos faz com que a ansiedade aumente. A estratégia de ensino utilizada pelo supervisor dando muita ênfase ao erro do aluno pode elevar ainda mais este estado apresentado pelo estagiário e colaborar com o movimento destrutivo (Gabriades, 2008). O acadêmico sente-se menos fragilizado quando escuta de seus colegas experiências que demonstram as dificuldades presentes no início do estágio, principalmente relacionadas à condução da prática (Guedes, 2006).

Quanto ao supervisor de estágio, este tem que se ater a aspectos importantes do desenvolvimento profissional dos alunos, para que se apropriem de teorias e técnicas de forma coerente e integrada, com o intuito de explorar os aspectos da subjetividade incluída no processo de aprendizagem dos alunos (Pires, 2011). A supervisão faz-se necessária em uma formação para além da técnica, pois favorece condições de mediação, oportunizando um espaço de fala e de ressignificações para os estagiários (Santos \& Nóbrega, 2017).

A relação entre aluno e supervisor é de extrema importância para que o estágio se desenvolva de forma satisfatória para a boa qualidade de ensino (Gabriades, 2008). Ao assumir o papel de apoio para o estagiário, o supervisor demonstra que está disponibilizando um suporte emocional e profissional, o que contribuirá para que o aluno tenha tranquilidade para desenvolver as suas atividades de forma adequada (Pires, 2011).

O aluno, na posição de aprendiz, necessita de tempo para poder controlar suas reações e, indiretamente, seus sentimentos relacionados a situações de estágio como um evento em si, bem como entender e aceitar suas dificuldades. Do outro lado, o professor fica, assim, empenhado num trabalho inicial, com a sensação de ser interminável e cansativo, mas também o percebe como gratificante, fascinante e desafiador, reiniciando-se, a cada novo semestre letivo e com cada aluno que inicia sua prática (Rudnicki \& Carlotto, 2007).

O aluno deve receber significativa atenção, quanto à orientação e apoio às dificuldades emocionais, com intuito de prepará-lo para o enfrentamento das possíveis dificuldades que surgem em decorrência desta primeira experiência em campo de estágio hospitalar. É fundamental que o aluno seja acolhido em suas limitações e conflitos, sendo fundamental a implantação de programas de suporte psicológico aos alunos, com o intuito de ajudá-los a lidar com as situações de conflito referentes à vida acadêmica (Soeiro \& Souza, 2010).

\section{Informações necessárias para a ałuação}

O estágio não é apenas uma aprendizagem prática na qual o aluno/acadêmico deverá articular a teoria com a prática, mas sim um processo de construção da identidade e amadurecimento profissional, além de um momento de desenvolvimento de estratégias de enfrentamento saudáveis frente aos estressores típicos das profissões do campo da saúde. É o momento de desenvolvimento de competências interpessoais importantes para a vida pessoal e profissional com sérias repercussões para sua qualidade de vida e da população que é alvo de sua escolha profissional (Rudnicki \& Carlotto, 2007).

As relações entre os estagiários e seus campos de atuação são centrais para proporcionar aprendizagens (Santos \& Nóbrega, 2017). O psicólogo no hospital pode atuar como facilitador da comunicação entre os membros da equipe de saúde, pacientes e familiares, realizando uma interlocução entre o médico (ou outro profissional) e o paciente (Angerami-Camon, 2001). É importante incentivar o estagiário na manutenção da comunicação com o médico, com o intuito de obter informações e esclarecimentos a respeito dos procedimentos médicos e medicamentosos acerca do paciente e deixando também a abertura necessária para que o profissional de Medicina também esclareça questões relativas aos aspectos psicológicos do paciente (Guedes, 2006).

Outro fator importante a ser ressaltado é a possibilidade de o psicólogo interagir com a equipe de saúde e participar das reuniões para discussão de casos clínicos visando uma melhor compreensão da situação dos pacientes e auxiliando na conquista da confiança no trabalho 
desempenhado (Campos, 1985). O psicólogo exerce a função de redirecionar o olhar dos demais profissionais para a individualidade de cada paciente e para os aspectos subjetivos do sujeito, favorecendo o reconhecimento dos aspectos psicológicos presentes na doença e na relação com a equipe se saúde, colaborando, assim, para a humanização do atendimento (Bruscato, Benedetti \& Lopes, 2004).

$\mathrm{O}$ atendimento psicológico realizado a pacientes internados e seus familiares, costuma ser realizado individualmente no leito do paciente. O objetivo das intervenções irá de acordo com a demanda do paciente, variando entre amenizar angústias e ansiedades decorrentes do processo de internação e do diagnóstico, vínculo mãe-bebê, adesão ao tratamento, preparação para a cirurgia, auxiliar no enfrentamento de situações de morte e luto, dentre outras (Tonetto \& Gomes, 2005). Outra informação importante para o psicólogo que adentra na psicologia hospitalar é se ater a necessidade de evolução dos atendimentos no prontuário, ou seja todas as informações relativas ao paciente hospitalizado devem ser preenchidas no prontuário por todos os profissionais (Silveira, 2010).

Um fator importante a ser lembrado é o setting terapêutico, pois este no ambiente hospitalar é totalmente diferente do experimentado no consultório clínico (Ismael, 2005). O espaço físico não é privativo, sendo utilizado pelos diversos profissionais da saúde. Esse fator pode ocasionar a interrupção de um atendimento no leito a qualquer momento principalmente por médicos e enfermeiros que tem a sua rotina ligada a horários específicos de intervenções a serem realizadas com o paciente (Almeida \& Malagris, 2011).

Diante desses aspectos, é essencial que o psicólogo tenha uma postura flexível com o objetivo de contornar as dificuldades e reconhecer que seu trabalho sofrerá interrupções, adiantamentos e cancelamentos necessários decorrentes da dinâmica hospitalar e que estes estarão fora do seu controle. Além de tudo isso é essencial que o psicólogo conheça a doença, a evolução e o prognóstico do seu paciente (Ismael, 2005; Romano, 1999).

Além de todos esses fatores é importante atentar-se para o momento em que o seu paciente receberá alta hospitalar, pois cabe ao psicólogo analisar a necessidade da continuidade do tratamento e tomar as condutas necessárias para a continuidade na própria instituição ou o encaminhamento para outro serviço (Tonetto \& Gomes, 2007).

\section{Considerações Finais}

Diante do que foi apresentado é possível observar que apesar do preparo realizado pelo estagiário durante o percurso acadêmico, ao se deparar com a prática muitas fantasias e inseguranças se tornam presente. Esse fato pode prejudicar ou auxiliar o estagiário, tudo dependerá da forma como este lidará com essas questões.

Além de fatores necessários para um bom desempenho do estágio em psicologia hospitalar muito ligado à interlocução da teoria e da prática, há também as inter-relações que o estagiário irá se deparar durante esse percurso. Decorrente disso é de extrema importância que ele tenha um ambiente acolhedor e propício para o seu crescimento pessoal e profissional, ambiente este que deverá ser proporcionado tanto pelo espaço físico quanto pelos profissionais que estão a sua volta.

O seu contato com o supervisor será de extrema importância nesse processo para que este estagiário se sinta acolhido e principalmente confiante no seu trabalho, pois é por meio do supervisor que espelhará seu trabalho e sua determinação para o desenvolvimento de uma prática cada vez melhor.

\section{Referências Bibliográficas}

Almeida, R. A.; \& Malagris, L. E. N. (2011). A prática da psicologia da saúde. Revista da Sociedade Brasileira de Psicologia Hospitalar, 14(2), 183-202.

Andrade, A. S.; Tiraboschi, G. A.; Antunes, N. A.; Viana, P. V. B. A.; Zanoto, P. A. \& Curilla, R. T. (2016). Vivências de graduandos em Psicologia. Psicologia: Ciência e Profissão, 36(4), 831-846.

Angerami-Camon, V. A. (2001). E a psicologia entrou no hospital. São Paulo: Pioneira.

Bruscato, L. W.; Benedetti, C.; \& Lopes, S. R. de A. (2004). A prática da psicologia hospitalar na santa casa de São Paulo: novas páginas em uma antiga história. São Paulo: Casa do Psicólogo.

Campos, T. C. P. (1985). Psicologia hospitalar: a atuação do psicólogo em hospitais. São Paulo: EPU.

Carvalho, M. T. M.; \& Sampaio, J. R. (1997). A formação do psicólogo e as áreas emergentes. Psicologia Ciência e Profissão, 17(1), 14-19.

Comin, F. S.; Souza, L. V. \& Santos, M. A. (2008). Tornar-se psicólogo: Experiência de estágio de Psico-oncologia em equipe multiprofissional de saúde. Revista Brasileira de Orientação Profissional, 9(2), 113-125.

Cury, B. de M. \& Neto, J. L. F. (2014). Do Currículo Mínimo às Diretrizes Curriculares: os estágios na formação do psicólogo. Psicologia em Revista, 20(3), 494-512.

Franco, S. L. R. (2001). Estudantes de Psicologia, eficácia adaptativa e a psicoterapia como medida preventiva em saúde mental. Mudanças - Psicoterapia e Estudos Psicossociais, 9(16), 41-63.

Gabriades, R. H. C. N. (2008). O significado da experiência dos primeiros atendimentos clínicos para os estagiários de um curso de psicologia de uma universidade particular na cidade de São Paulo. Dissertação de Mestrado, PUCSP, São Paulo.

Gorayeb, R. (2010). Psicologia da Saúde no Brasil. Psicologia: Teoria e Pesquisa. 26(spe): 115-122.

Guedes, C. R. (2006). A supervisão de estágio em psicologia hospitalar no curso de graduação: relato de uma experiência. Psicologia: ciência e profissão. 26(3), 516-523.

Ismael, S. M. C. (2005). A inserção do psicólogo no contexto hospitalar. In: Ismael S. M. C. (org). A prática psicológica e sua interface com as doenças (pp. 17-36). São Paulo: Casa do Psicólogo. 
Menezes, R. L. C.; \& Medrado, B. P. (2013). FORMAÇÃO EM PSICOLOGIA CLÍNICA: o estágio supervisionado como atividade potencial de desenvolvimento profissional. InterScientia, 1(2), 37-51.

Moulin, M. G. B. (1998). Trabalho, saúde mental e gênero - o caso das bancárias. Jornal Brasileiro de Psiquiatria, 47(4), 169-177.

Pires, V. S. (2011). O processo de subjetivação profissional durante os estágios supervisionados em psicologia. Dissertação de Mestrado, Universidade Federal de Uberlândia, Minas Gerais.

Romano, B. W. (1999). Princípios para a prática da psicologia clínica em hospitais. São Paulo: Casa do Psicólogo.

Rudnicki, T. \& Carlotto, M. S. (2007). Formação de estudante da área da saúde: reflexões sobre a prática de estágio. Revista da Sociedade Brasileira de Psicologia Hospitalar, 10(1), 97-110.

Santos, M. P (2015). Reflexões sobre a atuação do psicólogo no hospital. Trabalho de Conclusão de Curso (Graduação em Psicologia). Universidade Estadual da Paraíba, Campina Grande.

Santos, F. M. S. \& Jacó-Vilela, A. M. (2009). O psicólogo no Hospital Geral: estilos e coletivos de pensamento. Paideia, 19(43), 189-197.

Santos, L. J. (2011). Formação e atuação do psicólogo nos hospitais e maternidades do estado de Sergipe. Dissertação de Mestrado, Universidade Federal de Sergipe, Sergipe.
Santos, A. C. \& Nóbrega, D. O. (2017). Dores e Delícias em ser Estagiária: o Estágio na Formação em Psicologia. Psicologia: Ciência e Profissão, 37(2), 515-528.

Silva, J. A. P.; Coelho, M. T. A. D. \& Pontes, S. A. (2017). Psicanálise e Universidade: A Experiência do Estágio em Psicologia Clínica com Orientação Psicanalítica. Revista Psicologia, Diversidade e Saúde, 6(1), 44-49.

Silveira, A. M. V. (2010). Estudo do campo da psicologia hospitalar calcado nos fundamentos de gestão: estrutura, processos e resultados. Dissertação de Mestrado, Universidade Católica de Minas Gerais, Minas Gerais.

Soeiro, R.L. \& Souza, A. C. (2010). A relação entre o início da vivência no campo de prática hospitalar e o desenvolvimento de sintomas de distúrbios psicossomáticos em graduandos de enfermagem. Revista de Pesquisa: Cuidado é Fundamental Online, 2(supl): 438-40.

Tonetto, A. M.; \& Gomes, W. B. (2007). Competências e habilidades necessárias à prática psicológica hospitalar. Arquivos Brasileiros de Psicologia, 59(1), 38-50.

Tonetto, A. M. \& Gomes, W. B. (2005). Prática psicológica em hospitais: demandas e intervenções. Psico, 36(3), 283-291.

Torezan, Z. F.; Calheiros, T. C.; Mandelli, J. P. \& Stumpf, V. M. (2013). A Graduação em Psicologia prepara para o trabalho no hospital? Psicologia: ciência e profissão, 33(1), 132-145.

Submetido em: 19-07-2017

Aceito em: 1-11-2017 